University of Nebraska - Lincoln

DigitalCommons@University of Nebraska - Lincoln

\title{
The Role of the Clinical Nurse Specialist in the Future of Health Care in the United States
}

Jacqueline M. Gordon

Penn State Hershey Medical Center

Jennifer D. Lorilla

Tripler Army Medical Center

Cheryl A. Lehman

University of Texas Health Science Center, lehmanc@uthscsa.edu

Follow this and additional works at: https://digitalcommons.unl.edu/usarmyresearch

Gordon, Jacqueline M.; Lorilla, Jennifer D.; and Lehman, Cheryl A., "The Role of the Clinical Nurse Specialist in the Future of Health Care in the United States" (2012). US Army Research. 196.

https://digitalcommons.unl.edu/usarmyresearch/196

This Article is brought to you for free and open access by the U.S. Department of Defense at DigitalCommons@University of Nebraska - Lincoln. It has been accepted for inclusion in US Army Research by an authorized administrator of DigitalCommons@University of Nebraska - Lincoln. 


\title{
The Role of the Clinical Nurse Specialist in the Future of Health Care in the United States
}

\author{
Jacqueline M. Gordon, MSN, RN, CCNS, CCRN ${ }^{a}$, \\ Jennifer D. Lorilla, MSN, RN, United States Army Nurse Corps ${ }^{\mathrm{b}}$, \\ Cheryl A. Lehman, PhD, RN, CNS-BC, RN-BC, CRRN ${ }^{\mathrm{C}, *}$
}

\section{KEYWORDS}

- Clinical nurse specialist • CNS • APRN • Licensed independent practitioner

- Licensed independent provider

\section{KEY POINTS}

- The needs of the health care system and expectations of health care consumers can be met by an exceptionally trained Advanced Practice Registered Nurse (APRN): the clinical nurse specialist (CNS).

- However, role confusion related to the CNS, by developing competing roles that do not have APRN privileges, has created challenges for current and future CNSs to overcome.

- CNSs in the United States have consistently documented outcomes including improved quality of care, decreased costs, and improved patient satisfaction.

\section{INTRODUCTION}

The Clinical Nurse Specialist (CNS) has the potential to play a large and important role in assuring the delivery of high-quality health services to the citizens of the United States, but has often struggled with attaining recognition, reimbursement, and regard as an Advanced Practice Registered Nurse (APRN). The health care industry does not always seem to understand the value and potential of the CNS, in part attributable to the varied roles the CNS may play, such as independent licensed provider, clinical

The views expressed in this abstract/manuscript are those of the author(s) and do not reflect the official policy or position of the Department of the Army, Department of Defense, or the US Government.

a Heart and Vascular Institute, Penn State Hershey Medical Center, Hershey, PA 17033, USA;

b Department of Medicine, Tripler Army Medical Center, 1 Jarrett White Road, Tripler AMC, Hawaii 96859, USA; ' Department of Health Restoration and Care Systems Management, School of Nursing, The University of Texas Health Science Center at San Antonio, MC 7975, 7703 Floyd Curl Drive, San Antonio, TX 78229-3900, USA

* Corresponding author.

E-mail address: lehmanc@uthscsa.edu 
expert and nurse educator. Some markets limit the CNS to a single role (typically, the nurse educator), whereas others use the CNS to their fullest potential as clinical expert, involving their CNS employees in quality monitoring and improvement, implementation of evidence-based practices in the workplace, and as expert clinical practitioners in varied settings including primary, secondary, and tertiary care. This article explores the future of the CNS in health care in the United States.

\section{HISTORICAL PERSPECTIVE}

The CNS is 1 of 4 recognized APRN roles in the United States: the CNS, the Nurse Practitioner (NP), the Certified Nurse Midwife (CNM), and the Certified Nurse Anesthetist (CRNA). Under state-specific rules and regulations, the CNS has the ability and training to medically diagnose and manage the patient as an independent licensed provider; to prescribe medications, including scheduled drugs; to work as an expert nurse within systems to monitor and improve the quality of care; to provide training to practicing nurses and other providers; to collaborate with and/or lead the interprofessional team in team efforts such as program development; and to attend to the myriad of clinical patient, nurse, and systems issues that permeate the everyday world of the health care institution.

The use of the term "specialist" in nursing emerged in the 1900s. An article published in the first issue of the American Journal of Nursing entitled "Specialties in Nursing" noted the importance of specialty practice amongst nurses. ${ }^{1}$ Psychiatric nursing was the first nursing specialty; originating from the Quaker reformers who challenged the brutal treatment of the insane and advocated for gentler methods of social control in the second half of the nineteenth century. ${ }^{2}$ The CNS role grew substantially in the following decades. The American Nurses Association (ANA) officially recognized the CNS as an expert practitioner in 1974 and included master's education as a requirement for the CNS. ${ }^{2}$ The first state to recognize diagnosis and treatment as part of the scope of practice of specialty nurses was Idaho in $1971 .^{2}$ At this time boards of medicine and nursing met and developed regulations for practice. ${ }^{3}$

In the 1980s, requirements were instituted for nurses wishing to assume the title of CNS. The ANA's Social Policy Statement stated that to use the title CNS, study and supervised clinical practice must occur at the graduate level of education and requirements should be met for specialty certification through nursing's professional society. ${ }^{4}$ In the early 1980s, CNSs were sought after and new training programs were being demanded. By 1984 the National League for Nursing had accredited 129 programs for preparation of the CNS. ${ }^{2}$ By the end of the 1980s and beginning of the 1990s cost containment became a priority of the health care industry, and CNSs shifted away from being bedside expert clinicians toward administrator roles or staff educators, as a result of budget cutbacks. ${ }^{2}$ However, in 1995 the National Association of Clinical Nurse Specialists (NACNS) was established, and CNSs were subsequently identified for Medicare reimbursement eligibility in $1997 .{ }^{2}$ Organizational development of CNSs and legal inclusion of the CNS in reimbursement during the 1990s were vital to the continuation of the CNS role.

The CNS has regained support in recent years because of the noted contribution of the CNS to health care systems. The Institute of Medicine (IOM) has released reports focusing on the need for increased quality and safety in health care. ${ }^{5}$ The CNS plays a pivotal role in quality improvement, patient safety, and improved health care outcomes, and continues to deserve a place in the future of health care in the United States. 


\section{DEFINING THE CNS}

The NACNS has released numerous publications regarding the CNS role. ${ }^{6,7}$ The NACNS's Statement on Clinical Nurse Specialist Practice and Education ${ }^{7}$ clearly defines the role of the CNS. Historically the CNS role has been further divided into subroles such as direct patient care provider, consultant, educator, researcher, collaborator, and clinical leader 8 ; however, it was determined that this framework for CNS practice did little to distinguish the CNS from other nursing roles. ${ }^{9}$ NACNS defines CNSs as:

Licensed registered professional nurses with graduate preparation...from a program that prepares CNSs. They may also be prepared in a post-master's certificate program that is recognized by a national nursing accrediting body as preparing graduates to practice as a CNS for a specialty population...CNSs are clinical experts in the diagnosis and treatment of illness, and the delivery of evidence-based nursing interventions...CNSs work with other nurses to advance their nursing practices and improve outcomes, and provide clinical expertise to effect system-wide changes to improve programs of care. ${ }^{7}$

CNS areas of specialization can be based on population (eg, pediatric), type of problem (eg, surgical), setting (eg, inpatient), type of care (eg, critical care), or disease/pathology/medical specialty (eg, spinal cord injury, cardiac, pulmonary). ${ }^{7}$ CNS practice competencies are applied to 3 interacting spheres of influence: the patient/client sphere, the nurses/nursing sphere, and the organization/system sphere. ${ }^{7}$ CNS practice then occurs within the specialty chosen by that particular CNS in which core competencies are enacted.

\section{CNS COMPETENCIES}

CNSs enact the following 8 core competencies regardless of specialty or setting. ${ }^{7}$

1. Using "knowledge of differential illness diagnoses and treatments in comprehensive, holistic assessments of patients within the context of disease, diagnoses, and treatments."

2. Providing innovative interventions to achieve quality, cost-effective nurse-sensitive outcomes by designing, implementing, and evaluating individual and/or population-based programs.

3. Serving "as a leader/consultant/mentor/change agent in advancing the practice of nursing among other nurses and across organizations to achieve outcomes."

4. Advancing nursing practice by applying evidence-based interventions, using best-practice guidelines, and modifying professional standards and policies that direct care of nursing personnel and other providers of health care to improve outcomes.

5. Acting as a leader for interprofessional groups to facilitate collaboration with other disciplines in the attainment of outcomes across the continuum of care.

6. Identifying resource needs at the system level for delivery of nursing care, and attaining those resources.

7. Helping to "expand the practice of nursing through ongoing generation and acquisition of scientific knowledge and skills to maintain expert clinical competencies that leads to desired outcomes."

8. Demonstrating "professional citizenship and fiscal responsibility in the health care system by focusing on health policy and/or resource management to ensure quality, cost-effective outcomes of nursing care." 


\section{CNS EDUCATION}

CNS training, as already mentioned, is at the master's level. It includes courses in advanced pathophysiology, advanced pharmacology, and advanced health assessment, as well as courses in medical diagnosis and management, CNS role, nursing theory, research, and evidence-based practice.

In addition to the core requirements, NACNS recommends that the following additional core content specific to CNS practice be included in CNS educational programs:

- Theoretical foundations for CNS practice

- Theoretical and empiric knowledge of phenomena of concern that forms the basis for assessment, diagnosis, and treatment of illness and wellness

- Theoretical and scientific base for the design and development of innovative nursing interventions

- Clinical inquiry/critical thinking with advanced knowledge

- Selection, use, and evaluation of technology/products/devices

- Theories of teaching, mentoring, and coaching in all 3 spheres

- Influencing change

- Systems thinking

- Leadership development for interprofessional collaboration

- Consultation theory

- Measurement

- Evidence-based practice and research use ${ }^{7}$

CNS programs of study also include clinical experiences, with a preceptorship that includes at least 500 clinical hours. Ensuring that CNSs are educated based on the curriculum outlined by the NACNS is essential to producing well-prepared CNSs upon entry into practice.

\section{CURRENT STATE: THE CNS AND SOCIETAL NEEDS}

CNSs have the ability and training to function in several roles, including those as provider of patient care at an advanced level (ie, independent licensed provider) and as an advanced practice nurse within a health care system, educating staff, ensuring quality, and monitoring care. Patients, nurses, and health care institutions all have needs that the CNS can address. These needs include, among others: improving access to care for all populations; ensuring the delivery of high-quality, safe health care; meeting requirements of accrediting agencies; evaluating the best evidence and putting it into practice; educating nursing students and nurses; procuring safe, effective, and efficient equipment for patient care; and developing health care programs to meet population needs.

\section{Independently Licensed Provider Role}

With the Affordable Care Act (ACA) being signed into law in March 2010, the opportunity for transforming health care in the United States is both imminent and ongoing. The prospect of providing health care that is of higher quality, safer, more affordable, and more accessible is appealing. However, it is suspected that with the passage of the ACA an additional 32 million previously uninsured Americans will be seeking health care services. ${ }^{10}$ Who will care for these newly insured patients? Access to care has been an established long-term issue in the United States and has increased since the advent of the ACA. Physicians cannot carry the sole burden of caring for the 
increasing number of patients. APRNs such as CNSs can help bridge the gap and aid people in accessing health care in the United States if CNSs are allowed to practice to their fullest ability.

In the 1990s, federal regulations were enacted allowing Medicare reimbursement for CNSs that had been previously omitted. ${ }^{2}$ CNSs are now practicing around the nation in a multitude of environments such as primary care clinics, intensive care units, and specialty clinics, and are able to bill for their services. At present, scope of practice including prescriptive authority is regulated by each specific state Board of Nursing (BON). Each state is able to mandate restrictions on CNS practice. Some of these limitations include geographic distance between supervising physician and CNS, number of charts a supervising physician must review on a monthly basis, and number of advanced practice nurses (eg, CNSs and NPs) per supervising physician. ${ }^{10}$

For those CNSs practicing as independently licensed providers who bill Medicare, Medicare's definition of a CNS for billing purposes states that a CNS is:

- A registered nurse (RN) currently licensed to practice in the State where he or she practices and is authorized to furnish the services of a CNS in accordance with state law

- Has a Master of Science in Nursing or Doctor of Nursing Practice (DNP) in a defined clinical area of nursing from an accredited educational institution

- Is certified as a CNS by a recognized national certifying body that has established standards for CNSs

Medicare will pay CNSs if they meet Medicare qualification requirements and the practice or facility accepts Medicare payments, which is reimbursed at $85 \%$ of the Medicare Physician fee schedule amount. Medicare also has inpatient hospital billing principles, which in part state that the billed services must be a physician service, medically necessary, within the CNS scope of practice, and documented as required. Thus, qualified CNSs can be helpful to the hospital's bottom line by billing for services delivered in the inpatient as well as the outpatient setting. ${ }^{11}$

Legislative restrictions related to APRN practice often create decreased access to care. In reality, in salary and benefits CNSs and all APRNs cost less than physicians. Preparing an APRN in comparison with a physician requires less time and less expense, and education is less lengthy and less expensive than that of physicians. The IOM recommended in 2011 in the report The Future of Nursing: Leading Change, Advancing Health that APRNs should be able to practice to the full extent of their education and training. ${ }^{12}$ The IOM recommended actions for Congress to take, consisting of expanding the Medicare program to include coverage of APRN services that are within the scope of practice under applicable state law, just as physician services are now covered. The report further recommends that state legislatures reform scope of practice regulations to conform to the National Council of State Boards of Nursing APRN model rules and regulations. By allowing APRNs to practice to the full extent of their education and training, access to health care and primary care barriers can be surmounted.

CNSs have been proved to provide care at a lower cost than care provided by physicians, and a high level of evidence supports use of the CNS role to decrease the costs of care. ${ }^{13}$ One recent randomized controlled trial identified that cost savings were achieved by substituting physicians with diabetes nurse specialists in caring for patients with diabetes. ${ }^{14}$ The CNS has a very important role to play in providing care as an independent licensed provider, especially with regard to underserved populations, and should certainly be used within this role to improve access to health care in the United States. 


\section{Institutionally Focused Roles}

In addition to providing a bridge to the gap of access to care, the CNS is essential within our health care institutions. Potential roles for the CNS within an institution include expert clinician, nurse educator, interprofessional team leader, consultant, quality monitor, and champion of evidence-based practice.

For example, facilities receiving Magnet designation through the American Nurses Credentialing Center (ANCC) are highly revered, and have been proven to provide high-quality nursing care and exceptional processes for the delivery of nursing care. Clinical practice change and sustained process improvement are foundations of Magnet designation, and CNSs have the ideal skill set to make these impacts occur at a systems level and to aid in obtaining and sustaining Magnet recognition. ${ }^{15}$

CNSs often serve as nurse educator within institutions, ensuring that staff is current in knowledge. As a nurse educator, the CNS does more than provide required education for staff. The CNS conducts needs assessments of staff, examining national trends in care of the patient population, the latest evidence in medical and nursing care, the latest equipment purchases, the recent quality issues, patient comments, and other quality data to plan educational interventions that meet the needs of both the staff and the patients. The CNS then conducts outcome studies related to the education provided: did it make a difference and how?

CNSs also participate in education to train new nurses. A recent editorial by Gerard $^{16}$ reflects on her experience as a student in a baccalaureate nursing program. Students were educated by master's and doctorally prepared CNSs using the unification model of education that combined teaching with clinical practice, research, and/or administration. Gerard learned to respect the CNS practitionerteachers and saw that nurses, medical interns, and residents recognized them as the "go-to" experts driven by their expert knowledge and high quality of practice. CNSs taught students to be a role model and to participate in multidisciplinary meetings to improve plans of care for patients and to participate in professional organizations and meetings with government officials; guided students through systems-level thinking to support a safe environment and promote a high quality of care; and involved them in acquiring knowledge, research, and evaluating evidence. While education by CNS faculty are certainly measurable as outcomes such as graduation rates and NCLEX (National Council Licensure Examination) scores, CNSs who teach in schools of nursing also make immeasurable differences in the students they teach.

In recent years, specialty programs such as heart failure clinics and palliative care teams have become more popular. CNSs are emerging as leaders in the specialty fields that include programs certified by The Joint Commission (TJC), such as stroke centers. The TJC has also added advanced institutional certifications for other specialties including diabetes, ventricular assist devices, chronic kidney disease, and hip fracture. The CNS is the ideal APRN to aid facilities in obtaining this type of recognition while providing clinical expertise in the specialty areas.

\section{CNS OUTCOMES}

Outcomes such as improved quality of care can be directly related to CNS practice. The Centers for Medicare and Medicaid Services (CMS) has proposed a new system of reimbursement for hospitals that uses a value-based purchasing strategy based on quality data for annual repayment. ${ }^{17}$ The CNS within an institution can be instrumental in instituting tracking measures and procedural changes to improve quality of care and obtain maximal reimbursement. ${ }^{18}$ CNSs can implement strategies using 
evidence-based practice to improve quality of care and decrease costs. Optimal outcomes are synonymous with CNS practice.

The literature abounds with examples of the differences that the CNS can make in today's health care environment. Reductions in cost, length of stay, patient complications, and rehospitalizations, increases in patient satisfaction and quality of life, successful formation of new programs, and attainment of quality goals have all been attributed to CNS practice. The difference is apparent not only in patient outcomes, but also manifests in staff-centered interventions such as back safety programs that reduce cost and staff injury ${ }^{19}$ and the facilitation of evidence-based practice. ${ }^{18}$

The literature review by Moore and McQuestion ${ }^{20}$ presents an excellent summary of the role of the CNS in chronic disease management. A sample of CNS outcomes in this review include decreased rehospitalizations, shorter lengths of stay, lower hospital costs, improved quality of life for patients, and improvements in patient function, all of which are valuable to health care institutions in today's financial picture. Mahler ${ }^{21}$ describes the CNS role in development of a geropalliative model of care at a large health care facility. The CNS's role on the team was to influence the practice of nursing staff, and to be a nursing mentor, consultant, and educator.

Another example of CNS outcomes is the Cancer Reform Strategy, which recognized the importance of the CNS in improving the experience of patients diagnosed with cancer. ${ }^{22}$ The findings of this study demonstrated that CNS involvement improved patients' experience and satisfaction with the breast cancer service. As a result of the CNS role in the breast cancer service, the CNS had a visible role to patients as a consultant and was enabled to meet the informational needs of patients, assess their nursing needs, and support them through their treatment. The CNS's ability to coordinate care and provide appropriate and timely information, social and psychological support, and continuity of care enhanced the patients' experience through such a difficult time.

The Hospital of the University of Pennsylvania has operationalized unit-based CNSrelated outcomes into a CNS scorecard. This scorecard tracks outcomes at the unit level that can be influenced by the CNS, such as urinary tract infections, ventilatorassociated pneumonia, bloodstream infections, pressure ulcers, and falls, as well as staff knowledge levels, patient satisfaction, promotion of evidence-based practices, and staff turnover and satisfaction. ${ }^{23}$ The advantage of such a scorecard is that it allows the CNS to focus on issues important to the setting and the patients, and also communicates to others the importance of the CNS in affecting these outcomes.

\section{APPLICATION OF CNS PRACTICE}

Operationalizing the CNS role can be challenging. Two CNSs in the authors' own community are actively engaged in the CNS role at a local teaching hospital's intensive care unit (ICU). These CNSs have received accolades from the nurses they work with, physicians, and supervisors for their excellence in care and their importance to the organization. Recently they shared some examples of successful implementation of CNS practice.

The first CNS was able to change the culture in the medical ICU. Both physicians and nursing staff commented that before the CNS arrived, communication was poor and cohesiveness was absent. With his presence on daily interprofessional rounds, this CNS encouraged nurses on his unit to voice their concerns related to patient care, thus opening the door for better communication, improved teamwork, mutual respect, and collaboration among staff. He states that CNS presence empowered the nurses to believe that they could openly communicate with the team because 
they felt that the CNS was their advocate. Physicians are also appreciative that the CNS serves as consultant, expert clinician, and educator by providing on-the-spot training, while avoiding near misses, and improving patient outcomes through application of clinical knowledge and expertise.

Another example from this CNS concerns the introduction of an electromagnetic device used to safely insert feeding tubes at the bedside. Before implementation of the device, physicians were the only staff members allowed to place enteral feeding tubes for patients. After research and buy-in from the medical director and nurses, the CNS was able to develop and implement a clinical practice guideline that allowed nursing to place enteral feeding tubes after training, and 5 successful Dobhoff enteral feeding tube placements using the electromagnetic placement device. With the addition of a new and necessary nursing skill, the physicians were freed from placing feeding tubes and the nurses were able to add to their scope of practice.

The second CNS is currently involved in implementation of a mobility protocol, an evidence-based protocol to get ICU patients who meet criteria out of bed and walking while on a ventilator. This protocol encourages early mobility of ventilated patients and improves patient outcomes by increasing patients' well-being, function, and number of days out of bed while decreasing time spent on a ventilator and length of stay in the ICU and hospital, as well as decreasing delirium and dosages of narcotics and benzodiazepines. The mobility protocol was introduced by a physician, and the CNS served as a representative for nursing. She researched the nursing literature on mobility, and ensured that nursing concerns were addressed and incorporated into the protocol. She included bedside nurses in protocol development to ensure that the protocol was feasible and safe, and to get the initial buy-in. It has been almost 7 months since the initial trial. One of the nurses on the team recently attended the National Teaching Institute, a nursing educational conference, and attended all the lectures and posters that addressed mobility to evaluate their strategies and lessons learned; which has led to a mobility reinvigoration campaign. The CNS's goal is for nursing to own this protocol and spread this to other units for implementation.

This CNS is also involved in nursing research at the facility. She was consulted by a bedside nurse to assist with development of a study comparing penlight and pupillometer methods of pupil assessment. The CNS and the nurse searched together for articles on the subject matter and proceeded from there. The CNS also authored the Institutional Review Board protocol in keeping with the vision of the study. This study is still ongoing and is evaluating its outcomes.

Many CNSs in the authors' community are engaged in the role of independent licensed provider. Some of the sites are private clinics in a physician practice, such as vascular and cardiology, where they follow their own assigned patients. Others work on physician teams in ICUs, trauma clinics, and emergency rooms, where they see patients and also supervise the work of the medical residents. Some CNSs follow patients both in the clinic and in the hospital, assessing, diagnosing, and prescribing medications across the continuum of care. Some lead programs, such as heart failure clinics and stroke centers of excellence. Many of these CNSs serve as preceptors for CNS students, NP students, and even medical students.

All of these CNSs are recognized as key players in their facilities, and many are leaders at the national level. These CNSs highlight the importance of clinical leadership and commitment to patients, nursing, and the organization. CNSs demonstrate professional leadership in their active participation in mentorship and empowerment to the organization by working on patient-care issues to improve patient outcomes and costs for specialty populations. Their role enhances others' perception of their value. 


\section{THE COSTS OF CNS PRACTICE}

One key question for administrators is how to justify the cost of CNS practice. CNSs have done an excellent job of documenting the cost-effectiveness of their roles. The nurses and the institution benefit when length of stay is decreased; when complications of illness are prevented or recognized and treated early; when patients are satisfied and return to a practice or facility; when staff injury is prevented; when patients understand their disease and options for care; when cost-effective, safe, efficient equipment is selected and implemented; and when patient safety is addressed the patient. CNS cost-effectiveness is more than reimbursement from Medicare or an insurance company: it is often an intangible, difficult-to-measure benefit to their employer.

\section{CHALLENGES TO CNS PRACTICE}

Jones and Minarik ${ }^{24}$ report on the plight of the psychiatric CNS, a role being challenged through a societal shift to Family Psychiatric Nurse Practitioner. CNSs are also being challenged by the Clinical Nurse Leader (CNL) role, the DNP, and the ignorance of the health care system as to the value of a multiskilled CNS in comparison with roles such as the single-skilled educator.

The CNL in particular has the potential to confuse the employer. One of the basic differences between the CNL and the CNS is that the CNS is regarded as an APRN whereas the CNL has been labeled an "advanced generalist." The APRN designation specifically includes medical diagnosis and management and prescriptive authority, whereas the hallmarks of CNS practice, such as integrating evidence-based practice into health care, designing programs of care and innovative nursing interventions, and providing leadership and education to nurses, are not expressly included in the CNL role. The astute employer would take into consideration the broad range of talent of the CNS, and get "more bang for the buck" when hiring the CNS in a hospital role.

Consider the typical nursing unit. The most valued leaders have always been the senior, highly experienced RNs. These experienced leaders know the systems, how to manage (or get around!) them, and where the power lies, and also know their patient population very well. CNS designation is the next natural step for these experienced RNs: education at a higher level in the specific patient population, with added benefit from education in medical diagnosis and management, advanced pharmacology, advanced pathophysiology, advanced assessment, research, quality improvement, teaching and learning, and evidence-based practice. The CNS can then support other experienced RNs with education, system interventions, and higher-level skills needed to make a unit run well with the ability to offer a very high level of care. The CNS knows the patient population, and can clinically assist the nurses with complicated patients, high-level assessments, and development of plans for care and related interventions. The CNS becomes the "go-to" nurse.

Not all states recognize the CNS as a protected title or as an APRN, which then, in those states, allows nurses to work in the CNS role without special education or certification. Hudspeth ${ }^{25}$ sounded the alarm on the Consensus Model for APRN regulation and its impact on CNS employment. He noted that when the consensus documents are fully implemented, there will be a "large scale disenfranchisement of those nurses who hold graduate degrees in nursing, and who may have practiced in the role as a CNS for years, but who do not have any legal recognition allowing grandfathering, or who may be in a specialty that does not have sufficient numbers to maintain" a sound certification examination. 


\section{SUMMARY}

The United States health care system is currently faced with many challenges, including access to care, providing high-quality care, and cost containment. The ACA is adding to the burden on health care systems in the United States by increasing the number of patients who will be attempting to access care. Accrediting agencies including the TJC, ANCC, and CMS expect that providers and institutions will provide safe, cost-effective, quality care to all patients. The evident needs of the health care system and expectations of health care consumers can be met by an exceptionally trained APRN, namely the CNS. Some stakeholders, such as the IOM, have already recognized the potential of the CNS in helping to address society's health care issues. However, nursing's contribution to role confusion related to the CNS, by developing competing roles that do not have APRN privileges, has created challenges for current and future CNSs to overcome.

This review demonstrates that the CNS is multidimensional as an independent licensed provider, expert clinician, consultant, and educator. CNSs in the United States have consistently documented outcomes including improved quality of care, decreased costs, and improved patient satisfaction. Health care in the United States needs individuals prepared to be agents of change, to improve current processes and outcomes, and to promote an environment of ongoing interprofessional assessment and improvement of health care delivery processes. The CNS is poised to help accomplish these goals.

\section{REFERENCES}

1. Dewitt K. Specialties in nursing. Am J Nurs 1900;1:14-7.

2. Hamric AB, Spross JA, Hanson CM. Advanced practice nursing: an integrative approach. 4th edition. St Louis (MO): Saunders Elsevier; 2009.

3. Bullough B. The law and the expanding nursing role. Am J Public Health 1976;66: 249-54.

4. American Nurses Association. Nursing: a social policy statement. Kansas City (MO): American Nurses Association; 1980.

5. Institute of Medicine. Crossing the quality chasm: a new health system for the 21st century. Washington, DC: National Academy Press; 2001.

6. NACNS. A vision of the future for clinical nurse specialists. Harrisburg (PA): NACNS; 2007.

7. NACNS. Statement on clinical nurse specialist practice and education. 2nd edition. Harrisburg (PA): NACNS; 2004.

8. Hamric AB. Role development and functions. In: Spross J, Hamric AB, editors. The clinical nurse specialist in theory and practice. New York: Grune and Stratton; 1983. p. 39-56.

9. Fulton JS, Lyon BL, Goudreau KA. Foundations of clinical nurse specialist practice. New York: Springer; 2010.

10. CNAP. Texas needs a new approach to prescriptive authority. Available at: http:// cnaptexas.org/associations/9823/files/CNAP\%202013\%20Legislative\%20Initiative. pdf. Accessed May 6, 2012.

11. WOCN. Reimbursement of advanced practice registered nurse services: a fact sheet. J Wound Ostomy Continence Nurs 2012;39(Suppl 2):S7-16.

12. Institute of Medicine. The future of nursing: leading change, advancing health. Washington, DC: The National Academies Press; 2011.

13. Newhouse RP, Stanik-Hutt J, White KM. Advanced practice nurse outcomes 1990-2008: a systematic review. Nurs Econ 2011;29:230-51. 
14. Arts EE, Landewe-Cleuren SA, Schaper NC, et al. The cost-effectiveness of substituting physicians with diabetes nurse specialists: a randomized controlled trial with 2-year follow-up. J Adv Nurs 2012;68:1224-34.

15. Muller AC, Hujcs M, Dubendorf $P$, et al. Clinical nurse specialist practice and magnet designation. Clin Nurse Spec 2010;24:252-9.

16. Gerard P. Reinventing the role of the clinical nurse specialist as practitionerteacher to transform nursing education. Clin Nurse Spec 2010;24:277-8.

17. Charland K. Pay for performance comes to Medicare in 2009. Healthc Financ Manage 2007;61:60-4.

18. Tuite PK, George EL. The role of the clinical nurse specialist in facilitating evidence-based practice within a university setting. Crit Care Nurs Q 2010;33: 117-25.

19. Sedlak CA, Doheny MO, Jones SL, et al. The clinical nurse specialist as change agent: reducing employee injury and related costs. Clin Nurse Spec 2009;23: 309-13.

20. Moore J, McQuestion M. The clinical nurse specialist in chronic diseases. Clin Nurse Spec 2012;26:149-63.

21. Mahler $A$. The clinical nurse specialist role in developing a geropalliative model of care. Clin Nurse Spec 2010;24:18-23.

22. Hardie $H$, Leary $A$. Value to patients of a breast cancer clinical nurse specialist. Nurs Stand 2010;24:42-7.

23. Muller A, McCauley K, Harrington $P$, et al. Evidence-based practice implementation strategy: the central role of the clinical nurse specialist. Nurs Adm Q 2011;35: 140-51.

24. Jones JS, Minarik PA. The plight of the psychiatric clinical nurse specialist: the dismantling of the advanced practice nursing archetype. Clin Nurse Spec 2012;26:121-4.

25. Hudspeth $R$. Changes for the valuable clinical nurse specialist: a regulatory conundrum. Nurs Adm Q 2011;35:282-4. 